\title{
Group velocity measurement using spectral interference in near-field scanning optical microscopy
}

\author{
John D. Mills ${ }^{1}$, Tipsuda Chaipiboonwong ${ }^{1}$, Martin D.B. Charlton ${ }^{2,3}$, \\ Caterina Netti ${ }^{3}$, Majd E. Zoorob ${ }^{3}$, Jeremy J. Baumberg ${ }^{3,4}$ and William S. \\ Brocklesby \\ [1] Optoelectronics Research Centre, University of Southampton, SO17 1BJ, UK \\ [2] School of Electronics and Computer Science, University of Southampton, SO17 1BJ, UK \\ [3] Mesophotonics Ltd, Chilworth Science Park, Southampton, SO16 7NP, UK \\ [4] Dept of Physics and Astronomy, University Of Southampton, SOI7 IBJ, UK
}

\begin{abstract}
Near-field scanning optical microscopy provides a tool for studying the behavior of optical fields inside waveguides. In this experiment we measure directly the variation of group velocity between different modes of a planar slab waveguide as the modes propagate along the guide. The measurement is made using the spectral interference between pulses propagating inside the waveguide with different group velocities, collected using a near-field scanning optical microscope at different points down the guide, and spectrally resolved. The results are compared to models of group velocities in simple guides.
\end{abstract}


The use of near-field scanning optical microscopy (NSOM) to probe the fields inside planar optical waveguides is a technique that has been applied to many different systems. The availability of local information on sub-micron length scales can give much insight into how devices perform in the presence of imperfection, or in cases where modeling is difficult and not well-understood. NSOM has been applied in systems from simple $\mathrm{Y}_{\text {-junction }}$ couplers ${ }^{\mathrm{i}}$ to complex photonic crystal waveguides ${ }^{\mathrm{ii}}$. NSOM combined with modulation techniques has been used to image pulses propagating in rib waveguidesii, and recently in photonic crystal waveguides ${ }^{\mathrm{iv}}$, providing information about the group velocities by tracking the pulse within the guide.

In this paper we demonstrate a novel way to measure group velocity differences between different modes in a planar guide, using the broad spectrum of the femtosecond pulse together with the sub-micron collection area of an NSOM tip to advantage. In a guide which can support more than one mode, a launched pulse will typically couple into several different modes, which will each propagate at a different group velocity. As the pulses travel down the guide, they separate in time. An NSOM probe at some point along the guide can collect the evanescent field from many different modes, and once these fields are superposed, different spectral components interfere to produce spectral modulation. The time spacing of the pulses can be related directly to the modulation period of the spectral beating. Similar pulse interference techniques are used in coherent control experiments to deliberately produce a spectrally modulated pulse $\mathrm{v}^{\mathrm{v}}$. A Fourier transform of the spectrum recovers the time structure of the pulses as long as no significant changes in the spectral phase take place. Resolution of the spectral fringes depends in the local nature of the NSOM 
probe, as the spectral fringe pattern changes rapidly with pulse time separation and therefore with distance down the guide.

In this experiment, the waveguide used is a $500 \mathrm{~nm}$ high $\times 3500 \mathrm{~nm}$ wide slab of $\mathrm{Ta}_{2} \mathrm{O}_{5}$, on a layer of $\mathrm{SiO}_{2}$ grown on a silicon wafer, $6 \mathrm{~mm}$ long, produced by Mesophotonics Ltd. ${ }^{\mathrm{vi}}$. Effective index calculations indicate that $\sim 20$ modes can propagate within this structure, as the index of $\mathrm{Ta}_{2} \mathrm{O}_{5}$ is high $(\sim 2.1)$. Femtosecond pulses with pulse widths of $87 \mathrm{fs}$, wavelength $\sim 800 \mathrm{~nm}$, and energies of $2.1 \mathrm{~nJ}$ are launched into the guide. The evanescent field of the pulse is collected from the top surface of the waveguide using an NSOM tip consisting of a drawn single mode fiber with tip diameter $80 \mathrm{~nm}$, locked $20 \mathrm{~nm}$ from the surface of the guide using a shear force technique ${ }^{\mathrm{vii}}$. The decay length of the field into the air above the guide was measured to be $70 \mathrm{~nm}$. The output of the fiber tip is sent to a small spectrometer to record the spectrum at each position. The tip can be moved over $\sim 20 \mu \mathrm{m}$ distances using piezoelectric actuators, and can be moved along the guide using a micrometer.

Figure 1 shows various spectra of the pulse, measured by collecting light at $0.5 \mathrm{~mm}$ intervals along the central axis of the $\mathrm{Ta}_{2} \mathrm{O}_{5}$ slab with the NSOM tip. The effect of inter-modal interference on each acquired spectrum can be seen as modulation of the intensity versus wavelength. The spectral fringes appear irregular as they arise from several different inter-modal beats. However the expected narrowing of the spectral fringe separation as a function of distance along the guide can be seen clearly. The spectral fringe pattern moves in frequency because of the narrowing of the spectral fringe separation as the pulse time separation between different modes increases (as a function of spatial position along the guide). This results in the peaks of the pattern 
shifting by half their frequency separation over a distance of a few microns along the guide. Thus the fringes are only fully resolved if the light is collected using a local probe like an NSOM tip. In addition, the overall pulse spectrum broadens slightly as it propagates because of self-phase modulation ${ }^{\mathrm{vi}}$. The effect of this spread will be discussed in a later section.

In order to recover time information about the pulse separations which give rise to the spectral interference seen in figure 1, we can take the Fourier transform of the spectral intensity profile. The result of this is shown in figure 2. Several different linear variations of peak separation are obvious from figure 2 , and each of these corresponds to a particular group velocity difference between two modes of the guide. The time separation versus distance along guide is plotted in figure 3 for the best-resolved sets of peaks. The gradient gives the difference in group index $\left(\mathrm{c} / \mathrm{v}_{\mathrm{g}}\right)$ between different modes of the waveguide, in this case $0.0583,0.100,0.174$, and 0.258 .

No nonlinearity is necessary for this technique to give useful information. In this case, the presence of dispersion and nonlinear phase shifts in the guide complicates the interpretation of the Fourier transform of the spectral intensity, as all phase information is lost in the spectral intensity measurement. However, dispersion in this guide is relatively small, so for small values of the nonlinear phase shift the pulse spectral phase is still approximately constant. This allows the Fourier transform to be interpreted as reflecting the time structure of the pulse. At the pulse energy levels used for the data in figure 2, the maximum nonlinear phase at the peak of the pulse is $\sim \pi / 2$, from split-step beam propagation modeling of the spectral data. No variation in the position of the peaks in figure 2 is seen as the pulse energy is increased by a factor 
of $\sim 3$, indicating that the time structure is undistorted by this small nonlinearity. From simulations, the self-phase modulation affects the time structure at short times, as it changes the spectrum over broad frequency ranges, leaving the high-frequency spectral modulation responsible for the long time structure undistorted for small nonlinearities.

For comparison with this data, the group velocities of modes of the guide were calculated by both effective index and finite difference modeling, using the appropriate material dispersion. Tantalum pentoxide has significant variation in its index depending on exactly what the preparation method of the film is, but a Sellmeier-type equation for its index is given by Smith and Baumeister ${ }^{\text {viii }}$. The calculated group index difference between TM00 and TM01 is $\sim 0.1$ at $800 \mathrm{~nm}$, which corresponds well with the most obvious set of peaks in figure 2. Other modes are less obviously correlated to calculations. More accurate material dispersion values would increase the accuracy of the simulations, and the use of guides with fewer modes will enable a more rigorous test of theoretical calculations.

The peaks in figure 2 result from interference between different modes in the guide. Thus as the distribution of intensity between modes is changed, the interference will change. This is seen experimentally when changing the coupling of light into the guide. By scanning the input spot across the waveguide facet, a repeatable change in the intensities and time spacings of the mode differences can be seen, indicating that the initial mode distribution is changing as the coupling is varied, as expected. 
In summary, we have used the combination of spectral interference and local collection using an NSOM tip to provide direct information about group velocity differences between modes in a planar waveguide. Future work on guides with fewer modes is now in progress, so more accurate predictions can be made of the mode spectrum and distribution. 


\section{Figure Captions}

Figure 1 - Pulse spectrum measured at different points along the waveguide, and at its input and output (dotted lines). Spectral interference can be seen as modulation of the intensity versus wavelength. The initial $(0 \mathrm{~mm})$ and final $(6 \mathrm{~mm})$ spectra are not taken using the NSOM tip, but are integrated over the whole mode area.

Figure 2 - Fourier transform of spectra shown in figure 1. Several series of peaks with linearly-increasing time separation as a function of distance can be seen, each corresponding to the difference in group velocity between two particular modes.

Figure 3 - Time separation of different modes as a function of distance traveled down guide, from the Fourier transform of the spectral interference patterns. The gradients of these lines give the group velocity differences between modes. 
Figure 1

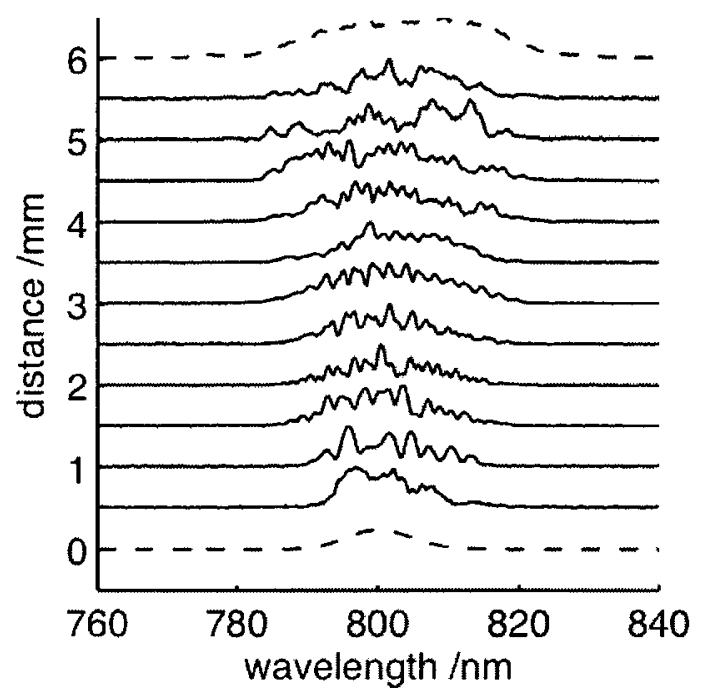

Figure 2

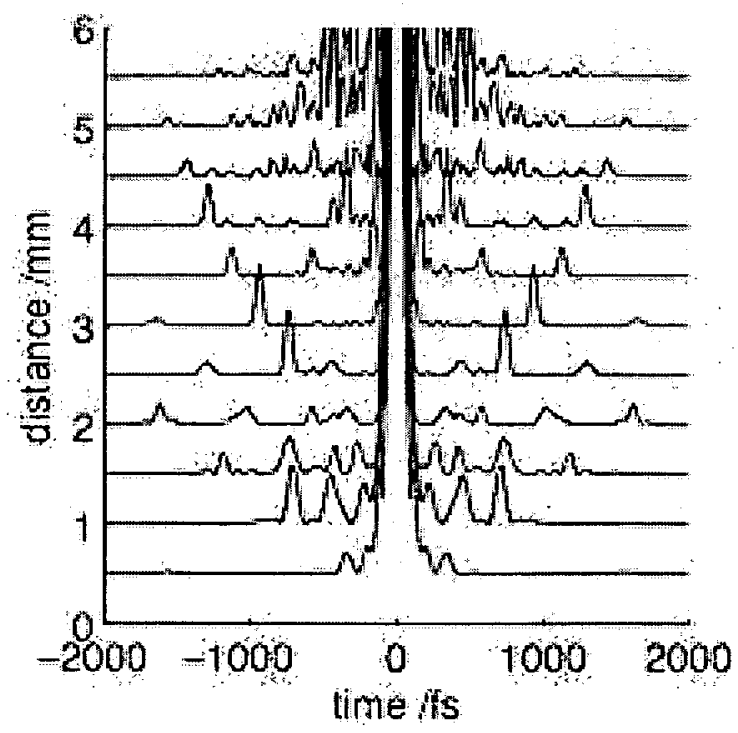


Figure 3

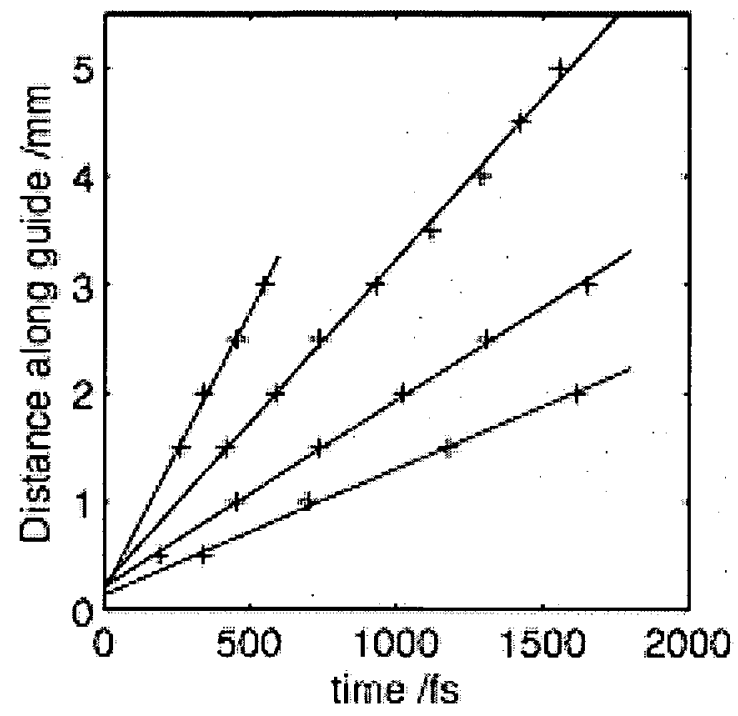




\section{References}

${ }^{i}$ D. P. Tsai, H. E. Jackson, R. C. Reddick, S. H. Sharp, and R. J. Warmack, Appl.

Phys. Lett. 56, 1515 (1990).

ii S. I. Bozhevolnyi, V. S. Volkov, T. Sondergaard, A. Boltasseva, P. I. Borel, and M.

Kristensen, Physical Review B 66, 235204 (2002).

${ }^{i i i}$ M. L. M. Balistreri, H. Gersen, J. P. Korterik, L. Kuipers, and N. F. van Hulst, Science 294 (5544), 1080 (2001).

${ }^{\text {iv }}$ R. J. P. Engelen, T. J. Karle, H. Gersen, J. P. Korterik, T. F. Krauss, L. Kuipers, and N. F. van Hulst, Optics Express 13, 4457 (2005).

${ }^{v}$ M. U. Wehner, M. H. Ulm, and M. Wegener, Opt. Lett. 22, 1455 (1997).

${ }^{v i}$ Caterina M. Netti, M. Zoorob, S. Roberts, M.D.B. Charlton, G.J. Parker, J.J.

Baumberg, J.R. Lincoln, F. Tauser, A. Zach, G.J. Flinn, and F. Lison, presented at the Commercial and Biomedical Applications of Ultrafast Lasers V, San Jose, CA, United States, 2005.

${ }^{\text {vii }}$ K. Karrai and R. D. Grober, Appl. Phys. Lett. 66, 1842 (1995).

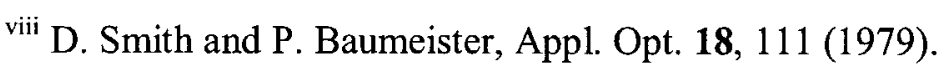

